\title{
High-resolution photocurrent spectroscopy of the positive trion state in a single quantum dot
}

\author{
J. D. Mar, ${ }^{1,2, *}$ J. J. Baumberg, ${ }^{2}$ X. L. Xu, ${ }^{1}$ A. C. Irvine,${ }^{3}$ C. R. Stanley,${ }^{4}$ and D. A. Williams ${ }^{1}$ \\ ${ }^{1}$ Hitachi Cambridge Laboratory, Cavendish Laboratory, Cambridge CB3 OHE, United Kingdom \\ ${ }^{2}$ NanoPhotonics Centre, Cavendish Laboratory, University of Cambridge, Cambridge CB3 OHE, United Kingdom \\ ${ }^{3}$ Microelectronics Research Centre, Cavendish Laboratory, University of Cambridge, Cambridge CB3 OHE, United Kingdom \\ ${ }^{4}$ Department of Electronics \& Electrical Engineering, University of Glasgow, Glasgow G12 8QQ, United Kingdom
}

(Received 15 February 2013; published 30 April 2013)

\begin{abstract}
We demonstrate high-resolution photocurrent spectroscopy of the positive trion state $\left(\mathrm{X}^{+}\right)$in a single InAs/GaAs self-assembled quantum dot (QD). Exploiting a Coulomb-mediated renormalization of the $\mathrm{X}^{+}$ transition energy, our approach allows for a sub- $\mu \mathrm{eV}$ resolution, which is more than four orders of magnitude higher than that in previous approaches. Furthermore, such high-resolution spectroscopy of $\mathrm{X}^{+}$permits us to obtain precise values for the $\mathrm{X}^{+}$permanent dipole moment and polarizability, by fitting a theoretical model to the measured transition energy as a function of electric field. Since optical initialization, manipulation, and readout of a QD spin rely on the trion state as an intermediary state, such high-resolution measurements of the $\mathrm{X}^{+}$spectrum and its dipole moment are essential for performing high-fidelity quantum computing operations on QD spin qubits.
\end{abstract}

DOI: 10.1103/PhysRevB.87.155315

\section{INTRODUCTION}

The spin of a carrier confined in a semiconductor quantum dot (QD) is a promising candidate for a qubit in quantum computing $(\mathrm{QC})$ due to its long relaxation and decoherence times. ${ }^{1-3}$ In order to perform QC operations, such QD spin qubits can be optically initialized, ${ }^{4,5}$ manipulated, ${ }^{6-8}$ and readout $^{9-11}$ by using as an intermediary state the ground-state trion-a singly charged exciton consisting of three carriers in the QD $s$-shell. Therefore, high-resolution spectroscopy of the trion state and a precise measurement of its dipole moment (i.e., Rabi frequency) are crucial for executing such QC operations with high fidelity. Photocurrent (PC) spectroscopy of the positive trion state $\left(\mathrm{X}^{+}\right)$, which consists of two heavy holes in a spin singlet and one electron of either spin in the QD $s$-shell, has been reported previously. ${ }^{11,12}$ However, the approach used in these works suffers from a considerably low resolution, which is determined by the spectral line width $(\sim 0.2 \mathrm{meV})$ of its transform-limited laser pulses. ${ }^{11,12}$

Here we demonstrate high-resolution PC spectroscopy of $\mathrm{X}^{+}$in a single InAs/GaAs self-assembled QD. Taking advantage of a Coulomb-mediated renormalization of the $\mathrm{X}^{+}$ transition energy by using a narrow-bandwidth $(\sim 1 \mathrm{MHz})$ continuous-wave (cw) laser for optical excitation of $\mathrm{X}^{+}$, our approach allows for sub- $\mu \mathrm{eV}$ resolution, which is limited only by the spectral line width of the laser and is more than four orders of magnitude higher than that in the previous works. ${ }^{11,12}$ Furthermore, such high-resolution spectroscopy of $\mathrm{X}^{+}$allows us to obtain precise values for the $\mathrm{X}^{+}$permanent dipole moment and polarizability in the vertical (growth) direction, by fitting a theoretical model to the measured transition energy as a function of vertical electric field.

\section{EXPERIMENTAL DETAILS}

\section{A. Photodiode device and PC experimental setup}

The device used throughout this work was designed for PC measurements on single QDs and fabricated as an $n-i$ Schottky photodiode structure based on a two-dimensional electron gas (2DEG). A single layer of InAs self-assembled QDs, which was grown to yield a low surface-density of QDs $\left(\sim 10^{9} \mathrm{~cm}^{-2}\right)$, is embedded in a 250 -nm-thick $i$-GaAs layer and located $50 \mathrm{~nm}$ above a $\mathrm{Si} \delta$-doped GaAs layer $\left(N_{d}=5 \times 10^{12} \mathrm{~cm}^{-2}\right)$, from which the 2DEG forms that is confined in the resultant V-shaped potential well. ${ }^{13,14}$ Submicrometer-sized apertures were etched into an $\mathrm{Al}$ shadow mask, which had been evaporated onto the Schottky contact, via electron-beam lithography and serve to isolate single QDs for our single-QD measurements. Finally, a AuGeNi ohmic contact was established to the 2DEG and $\mathrm{Cr} / \mathrm{Au}$ bond pads were fabricated on the ohmic and Schottky contacts to allow for electrical connection to a voltage source and current meter. At the QD layer, the vertical (growth-direction) electric field $F=\left(V_{i}-V_{b}\right) / d$, where $V_{i}$ is the intrinsic built-in (Schottky) potential, $V_{b}$ is the applied bias voltage between the 2DEG and Schottky contact, and $d$ is the distance between the $\delta$-doping and Schottky contact.

In the single-QD PC measurements performed throughout this work, resonant laser excitation was derived from two independently-tunable narrow-bandwidth $(\sim 1 \mathrm{MHz})$ externalcavity diode lasers in the Littrow configuration. After passing each through a half-wave plate, Glan-Thompson polarizer, and quarter-wave plate to control the intensity and circular polarization, the laser outputs were focused onto a device aperture using a microscope objective lens [numerical aperture $(\mathrm{NA})=0.5]$ mounted on a piezo-driven $x y z$-stage for precise nanopositioning. Experiments on the device were performed at low temperatures $(\sim 4.2 \mathrm{~K})$ in a He-flow cold-finger optical cryostat. $V_{b}$ and measurement of the PC were provided by a commercial source-measure unit (10 fA resolution) connected to the device via coaxial cables to the cryostat's electrical feedthroughs.

Further details of our device and PC experimental setup are found in Refs. 15-17 and Refs. 16 and 17, respectively.

\section{B. Scheme for $\mathrm{X}^{+} \mathrm{PC}$ measurement}

The scheme used here for PC measurements of $\mathrm{X}^{+}$is illustrated in Fig. 1. With the photodiode under reverse bias 


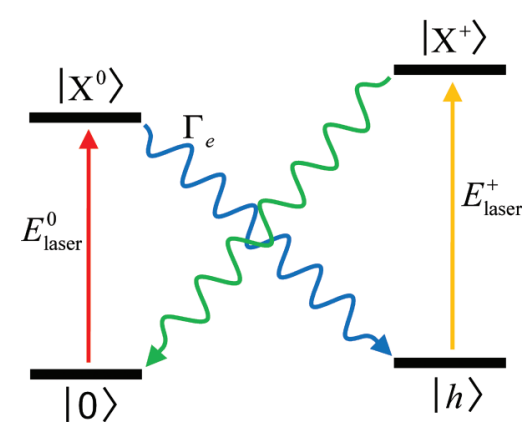

FIG. 1. (Color online) Scheme used for PC measurements of $\mathrm{X}^{+}$. $|0\rangle$ represents the QD initially empty of all carriers and $E_{\text {laser }}^{0}$ is the energy of a polarized cw laser field that resonantly excites $X^{0}$ in the QD. The $\mathrm{X}^{0}$ electron tunnels out at a rate of $\Gamma_{e}$ due to the electric field, thereby leaving a single hole in the QD denoted by $|h\rangle . E_{\text {laser }}^{+}$is the energy of a second cw laser field, which is cross-polarized with respect to the first laser, that resonantly excites $\mathrm{X}^{+}$in the QD. The subsequent tunneling-out of all carriers results in a measurable PC signal and a return to $|0\rangle$ for the next measurement cycle. Taking advantage of a Coulomb-mediated renormalization of the $\mathrm{X}^{+}$transition energy, our scheme uses narrow-bandwidth cw lasers in order to achieve $\mathrm{X}^{+} \mathrm{PC}$ spectroscopy with high resolution.

such that the QD $s$-shell (lowest-energy) electron level is well above the Fermi level in the 2DEG, the QD is initially empty of all carriers, as represented by $|0\rangle$ in Fig. 1. A polarized cw laser field with energy $E_{\text {laser }}^{0}$ resonantly excites in the QD $s$-shell a neutral exciton $\left(\mathrm{X}^{0}\right)$, which consists of a heavy hole and an electron with spins corresponding to the polarization of the laser field. In the presence of the electric field, this is followed by rapid ionization of $\mathrm{X}^{0}$ via electron tunneling at a rate $\Gamma_{e}$, since the effective mass of an electron is much smaller than that of a heavy hole, thereby leaving a single hole in the QD, denoted by $|h\rangle$ in Fig. 1, with a known spin that was determined by the laser polarization, assuming the absence of spin relaxation and $\mathrm{X}^{0}$ fine-structure splitting due to an anisotropic in-plane electron-hole exchange interaction. From state $|h\rangle$, the $\mathrm{X}^{+}$transition is then resonantly excited to create $\mathrm{X}^{+}$using a second cw laser field, which is cross-polarized with respect to the $\mathrm{X}^{0}$ laser, since the two heavy holes in the $s$-shell must form a spin-singlet, and has an energy $E_{\text {laser }}^{+}$that differs from $E_{\text {laser }}^{0}$ due to a renormalization of the $\mathrm{X}^{+}$transition energy as a result of additional Coulomb interactions in the $\mathrm{X}^{+}$few-particle state in the QD. The subsequent tunneling of all carriers out of the QD due to the electric field results in a measurable PC signal, which consists of a component due to the optical excitation of each of $\mathrm{X}^{0}$ and $\mathrm{X}^{+}$, and a return to the initial state $|0\rangle$ for the next measurement cycle. It is important to realize that, since $\mathrm{X}^{0}$ and $\mathrm{X}^{+}$have different transition energies due to their particular Coulomb interactions, the laser with an energy of $E_{\text {laser }}^{0}\left(E_{\text {laser }}^{+}\right)$optically excites only the $\mathrm{X}^{0}\left(\mathrm{X}^{+}\right)$transition. Taking advantage of this fact, our scheme therefore uses $\mathrm{cw}$ lasers, which have an orders-of-magnitude narrower spectral bandwidth compared to that of transform-limited pulsed lasers, ${ }^{11,12}$ in order to achieve high-resolution PC spectroscopy of $\mathrm{X}^{+}$.

\section{PC measurement of $\mathrm{X}^{+}$spectrum}

Using the scheme for $\mathrm{X}^{+} \mathrm{PC}$ measurements as described above (Fig. 1), the PC spectrum of $\mathrm{X}^{+}$is obtained as follows, where a narrow-bandwidth cw laser with an energy of $E_{\text {laser }}^{0}$ $\left(E_{\text {laser }}^{+}\right)$is tuned to the $\mathrm{X}^{0}\left(\mathrm{X}^{+}\right)$transition energy. With the two lasers cross-polarized, the $\mathrm{X}^{0}$ and $\mathrm{X}^{+}$transition energies are then swept through their respective laser energies roughly simultaneously via the quantum-confined Stark effect (QCSE). $\mathrm{X}^{0}$ excitation followed by fast ionization via electron tunneling allows for possible excitation of $\mathrm{X}^{+}$, resulting in a total PC signal consisting of an $\mathrm{X}^{0}$ and an $\mathrm{X}^{+} \mathrm{PC}$ component after all carriers have tunneled out of the QD. As the $\mathrm{X}^{+}$absorption peak is swept through $E_{\text {laser }}^{+}$, excitation of $\mathrm{X}^{+}$is dependent not only on the absorption strength of $\mathrm{X}^{+}$at $E_{\text {laser }}^{+}$, but also on the absorption strength of $\mathrm{X}^{0}$ at $E_{\text {laser }}^{0}$ for a given $V_{b}$. Therefore, the $\mathrm{X}^{+} \mathrm{PC}$ component as a function of $V_{b}, I_{+}\left(V_{b}\right)$, obtained by simply subtracting the $\mathrm{X}^{0} \mathrm{PC}$ component as a function of $V_{b}, I_{0}\left(V_{b}\right)$, from the total PC signal as a function of $V_{b}$, $I_{\text {total }}\left(V_{b}\right)$, does not yield an accurate PC measurement of the actual $\mathrm{X}^{+}$spectrum, especially when there is detuning between the transition energy and laser energy separations.

A PC measurement of the $\mathrm{X}^{+}$spectrum as a function of $V_{b}$ (i.e., energy $E$ ) is obtained by subtracting $I_{0}\left(V_{b}\right)$ from $I_{\text {total }}\left(V_{b}\right)$, then dividing the result by the $\mathrm{X}^{0}$ probability distribution. This can be shown as follows. We first define the $\mathrm{X}^{0}$ probability distribution as a function of $E$, for a given $V_{b}$, as a Lorentzian function $L_{0}\left(E, E_{c}^{0}\left(V_{b}\right), \gamma_{0}\right)$, where $E_{c}^{0}\left(V_{b}\right)=E_{c}^{0}(0)-\alpha_{0} V_{b}$ is the center of the Lorentzian peak as a function of $V_{b}$ and $\alpha_{0}$ is due to the QCSE, assuming that the Stark shift is linear which is a reasonable approximation throughout the $V_{b}$ range for the PC measurements, and $\gamma_{0}$ is the line width of the Lorentzian peak. We also define similarly the probability distribution for $\mathrm{X}^{+}$as $L_{+}\left(E, E_{c}^{+}\left(V_{b}\right), \gamma_{+}\right)$ with analogous parameters. The total PC signal is the sum of the $\mathrm{X}^{0}$ and $\mathrm{X}^{+} \mathrm{PC}$ components as a function of $V_{b}$; that is,

$$
I_{\mathrm{tot}}\left(V_{b}\right)=I_{0}\left(V_{b}\right)+I_{+}\left(V_{b}\right),
$$

where

$$
\begin{aligned}
& I_{0}\left(V_{b}\right)=e A_{0} L_{0}\left(E_{\text {laser }}^{0}, E_{c}^{0}\left(V_{b}\right), \gamma_{0}\right), \\
& I_{+}\left(V_{b}\right)=e A_{+} L_{0}\left(E_{\text {laser }}^{0}, E_{c}^{0}\left(V_{b}\right), \gamma_{0}\right) \cdot L_{+}\left(E_{\text {laser }}^{+}, E_{c}^{+}\left(V_{b}\right), \gamma_{+}\right),
\end{aligned}
$$

where $A_{0}$ is the photon absorption rate of $X^{0}$ when $E_{\text {laser }}^{0}$ is exactly resonant with $\mathrm{X}^{0}$ [i.e., $\left.E_{\text {laser }}^{0}=E_{c}^{0}\left(V_{b}\right)\right], A_{+}$is the photon absorption rate of $\mathrm{X}^{+}$when $E_{\text {laser }}^{+}$and $E_{\text {laser }}^{0}$ are exactly resonant with $\mathrm{X}^{+}$and $\mathrm{X}^{0}$, respectively [i.e., when $E_{\text {laser }}^{+}=$ $E_{c}^{+}\left(V_{b}\right)$ and $\left.E_{\text {laser }}^{0}=E_{c}^{0}\left(V_{b}\right)\right]$, and $e$ is the elementary charge. By subtracting $I_{0}\left(V_{b}\right)$ from $I_{\text {total }}\left(V_{b}\right)$, we are left with

$$
I_{+}\left(V_{b}\right)=e A_{+} L_{0}\left(E_{\text {laser }}^{0}, E_{c}^{0}\left(V_{b}\right), \gamma_{0}\right) \cdot L_{+}\left(E_{\text {laser }}^{+}, E_{c}^{+}\left(V_{b}\right), \gamma_{+}\right) \text {. }
$$

Therefore, the $\mathrm{X}^{+} \mathrm{PC}$ spectrum as a function of $V_{b}$ is defined as

$$
\begin{aligned}
e A_{+} L_{+}\left(E_{\text {laser }}^{+}, E_{c}^{+}\left(V_{b}\right), \gamma_{+}\right) & =\frac{I_{+}\left(V_{b}\right)}{L_{0}\left(E_{\text {laser }}^{0}, E_{c}^{0}\left(V_{b}\right), \gamma_{0}\right)} \\
& =\frac{I_{\text {tot }}\left(V_{b}\right)-I_{0}\left(V_{b}\right)}{L_{0}\left(E_{\text {laser }}^{0}, E_{c}^{0}\left(V_{b}\right), \gamma_{0}\right)} .
\end{aligned}
$$

That is, as stated earlier, the $\mathrm{X}^{+} \mathrm{PC}$ spectrum as a function of $V_{b}$ is obtained by subtracting the $\mathrm{X}^{0} \mathrm{PC}$ component (i.e., the 
PC spectrum of $X^{0}$ ) from the total PC signal, then dividing the result by the $\mathrm{X}^{0}$ probability distribution.

Finally, we point out that we have used the above method of tuning the exciton transition energies through their respective fixed laser energies, rather than the alternative method of tuning $E_{\text {laser }}^{+}$through $\mathrm{X}^{+}$while keeping $E_{\text {laser }}^{0}$ resonantly fixed on $\mathrm{X}^{0}$, because we are unable to tune $E_{\text {laser }}^{+}$through the entire $\mathrm{X}^{+}$absorption peak without tuning the laser beyond its rated mode-hop-free tuning range $(\sim 20 \mathrm{GHz})$, as will be seen in the experimental results of the $\mathrm{X}^{+} \mathrm{PC}$ spectrum. Furthermore, our method offers the advantage of greater stability during a PC measurement sweep, since fluctuations in laser intensity and alignment associated with tuning the laser energy are avoided.

\section{RESULTS AND DISCUSSION}

Initial measurements are performed using bias-dependent micro-photoluminescence ( $\mu$-PL) spectroscopy, via aboveband gap laser excitation using a $\mathrm{HeNe} \mathrm{cw}$ laser, in order to identify straightforwardly single QDs and the transition energies of their various excitonic states, which differ due to a renormalization of exciton transition energies as a result of the particular Coulomb interactions between charge carriers confined in the QD, under low electric-field conditions. ${ }^{15,17}$ For $\mathrm{X}^{+} \mathrm{PC}$ spectroscopy, since the exciton transition energies redshift with increasing electric field due to the QCSE, $E_{\text {laser }}^{0}=1364.37 \mathrm{meV}$ and $E_{\text {laser }}^{+}=1371.03 \mathrm{meV}$ are tuned slightly redshifted relative to the $\mathrm{X}^{0}$ and $\mathrm{X}^{+}$transition energies, respectively, and the $\mathrm{X}^{0}$ and $\mathrm{X}^{+}$transition energies are swept through their respective laser energies roughly simultaneously with increasing reverse bias, recalling that excitation of $\mathrm{X}^{+}$is conditional on the ionization of $\mathrm{X}^{0}$ via fast electron tunneling (Fig. 1). As shown in Fig. 2, by subtracting the PC component
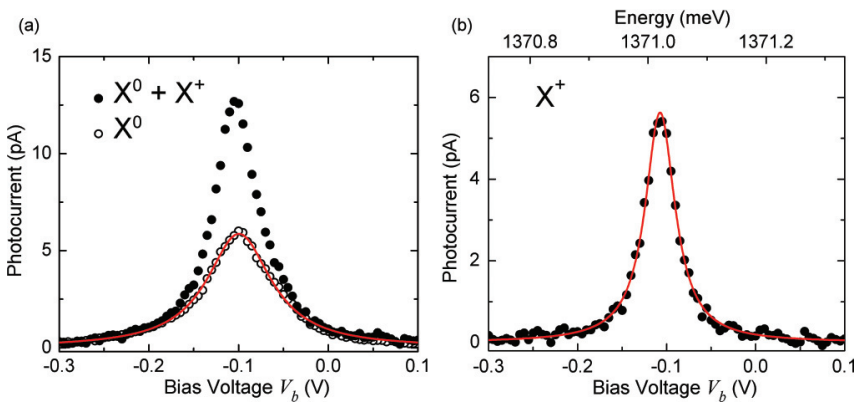

FIG. 2. (Color online) Experimental results for the PC measurement of the $\mathrm{X}^{+}$spectrum. Sweeping the $\mathrm{X}^{0}$ and $\mathrm{X}^{+}$transition energies through $E_{\text {laser }}^{0}=1364.37 \mathrm{meV}$ and $E_{\text {laser }}^{+}=1371.03 \mathrm{meV}$, respectively, we obtain an overall PC signal [closed circles in (a)], which consists of an $\mathrm{X}^{0}$ and an $\mathrm{X}^{+} \mathrm{PC}$ component. By subtracting the $\mathrm{X}^{0} \mathrm{PC}$ component [open circles in (a)] from the overall PC signal and then dividing the result by the $\mathrm{X}^{0}$ probability distribution, the $V_{b}$ spectrum of $\mathrm{X}^{+}$can be extracted [(b), bottom $x$-axis]. A Lorentzian fit curve [(b), solid line] yields a precise value for the $\mathrm{X}^{+}$peak $V_{b}$, thereby generating corresponding values for $V_{b}$ and $\mathrm{X}^{+}$transition energy of $-107.4 \mathrm{mV}$ and $1371.03 \mathrm{meV}$, respectively. The $\mathrm{X}^{0}\left(\mathrm{X}^{+}\right)$laser was chosen to be $\sigma^{+}\left(\sigma^{-}\right)$-circularly polarized. The energy spectrum of $\mathrm{X}^{+}[(\mathrm{b})$, top $x$-axis] is obtained from the $V_{b}$ spectrum of $\mathrm{X}^{+}[(\mathrm{b})$, bottom $x$-axis] through a precise relation that is used to convert between $V_{b}$ and $\mathrm{X}^{+}$ transition energy. due to $\mathrm{X}^{0}$ [Fig. 2(a), open circles] from the resulting overall PC signal [Fig. 2(a), closed circles], which is created due to the tunneling-out of all carriers, and then dividing by the $\mathrm{X}^{0}$ probability distribution to account for possible detuning between the transition energy and laser energy separations, a spectrum of $\mathrm{X}^{+}$can be extracted [Fig. 2(b)], as described earlier. Fitting a Lorentzian curve [Fig. 2(b), solid line] to the experimental data of the $\mathrm{X}^{+}$spectrum, a precise value for the $\mathrm{X}^{+}$peak $V_{b}=-107.4 \mathrm{mV}$ can be obtained. Therefore, we have determined corresponding values for $V_{b}$ and $\mathrm{X}^{+}$transition energy of $-107.4 \mathrm{mV}$ and $1371.03 \mathrm{meV}$, respectively. Notice the reduced line width of $\mathrm{X}^{+}$compared to that of $\mathrm{X}^{0}$ due to an increased Coulomb attraction for the electron in the $\mathrm{X}^{+}$ few-particle state. It is also worth noting that, from the relative PC peak amplitudes of $\mathrm{X}^{0}(5.86 \mathrm{pA})$ and $\mathrm{X}^{+}(5.63 \mathrm{pA})$, the probability is about $96 \%$ that an $\mathrm{X}^{+}$photon is absorbed while a single hole is still resident in the QD following $\mathrm{X}^{0}$ ionization. Therefore, given that the hole tunneling time is $\sim 2.9 \mathrm{~ns}$ at this $V_{b}$ (Ref. 16) and assuming an $\mathrm{X}^{0}$ fine-structure splitting of zero and the absence of hole-spin relaxation prior to tunneling, the rate of photon absorption is $\sim 119 \mathrm{ps}^{-1}$ subsequent to tunneling-out of the $\mathrm{X}^{0}$ electron, for an $\mathrm{X}^{+}$laser intensity on the sample of $\sim 1.7 \mu \mathrm{W}$. For the measurements in Fig. 2 and those presented throughout this work, the $\mathrm{X}^{0}\left(\mathrm{X}^{+}\right)$laser was chosen to be $\sigma^{+}\left(\sigma^{-}\right)$-circularly polarized. However, the same results were obtained when the $\mathrm{X}^{0}\left(\mathrm{X}^{+}\right)$laser was chosen to be $\sigma^{-}\left(\sigma^{+}\right)$circularly polarized, as expected since there is no applied magnetic field. Furthermore, while an in-plane anisotropic electron-hole exchange interaction does not exist for $\mathrm{X}^{+}$due to a total hole spin of zero, we would expect to obtain the same results also when the $\mathrm{X}^{0}$ and $\mathrm{X}^{+}$lasers are orthogonal in any polarization basis.

In order to convert an $\mathrm{X}^{+} \mathrm{PC}$ spectrum from a $V_{b}$ spectrum (Fig. 2, bottom $x$-axes) to an equivalent $\mathrm{X}^{+}$transition energy spectrum, the equivalent $\mathrm{X}^{+} \mathrm{PC}$ measurement described earlier in Fig. 2 (bottom $x$-axes) is performed for a series of distinct corresponding values of $E_{\text {laser }}^{0}$ and $E_{\text {laser }}^{+}$, as the $\mathrm{X}^{+}$absorption peak will appear at different values of $V_{b}$ for varying $E_{\text {laser }}^{+}$according to the QCSE. Therefore, using the resultant corresponding values of $V_{b}$ and $\mathrm{X}^{+}$transition energy, which is known from the corresponding values of $E_{\text {laser }}^{+}$, it will be possible to interpolate precise values for $\mathrm{X}^{+}$ transition energy as a function of $V_{b}$ by fitting a quadratic curve to the experimental data as a result of the QCSE. Accordingly, Fig. 3 presents $\mathrm{X}^{+} \mathrm{PC}$ spectra for a series of distinct values of $E_{\text {laser }}^{+}$, which are indicated alongside their corresponding spectrum, throughout the $V_{b}$ range within which a measurable $\mathrm{X}^{+} \mathrm{PC}$ signal was obtained. The spectra are shifted vertically with respect to each other for clarity. Each $\mathrm{X}^{+} \mathrm{PC}$ spectrum has a Lorentzian curve fit to it (Fig. 3, solid lines) in order to determine its peak $V_{b}$. Moving from high to low values of $V_{b}$ (i.e., for increasing reverse bias and $F$ ), we observe a gradual decrease in the $\mathrm{X}^{+} \mathrm{PC}$ peak amplitude that is due to an increasing tunneling rate of the $\mathrm{X}^{0}$ hole, while the $\mathrm{X}^{+}$laser intensity remains constant. The observed broadening of the $\mathrm{X}^{+}$line width, which also contributes to the decrease in peak amplitude, is the result of a decreasing $\mathrm{X}^{+}$ lifetime, in accordance with time-energy uncertainty, due to a rapidly increasing tunneling rate of the $\mathrm{X}^{+}$electron out of the QD. 


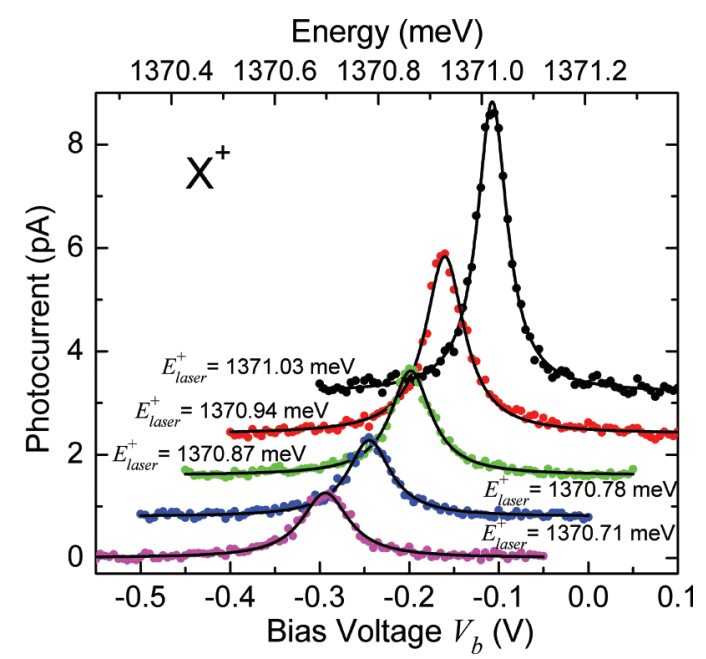

FIG. 3. (Color online) $\mathrm{X}^{+} \mathrm{PC}$ spectra for a series of distinct values of $E_{\text {laser }}^{+}$, which are indicated next to their corresponding spectrum, throughout the $V_{b}$ range within which a measurable PC signal can be obtained. The spectra are shifted vertically with respect to each other for clarity. Each spectrum has a Lorentzian curve fit to it (solid lines) in order to determine its peak $V_{b}$. The energy spectra of $\mathrm{X}^{+}$ (top $x$-axis) is obtained from the $V_{b}$ spectra of $\mathrm{X}^{+}$(bottom $x$-axis) through a precise relation that is used to convert between $V_{b}$ and $\mathrm{X}^{+}$ transition energy.

Figure 4 shows a plot of the $\mathrm{X}^{+}$transition energy as a function of $V_{b}$ (bottom $x$-axis) obtained through both PC and $\mu$-PL spectroscopy of $\mathrm{X}^{+}$in our single QD. While the data points in the $\mathrm{PC}$ regime were acquired by extracting values of the peak $V_{b}$ from Lorentzian curve fits to the $\mathrm{X}^{+} \mathrm{PC}$ spectra for known values of $E_{\text {laser }}^{+}$(Fig. 3), those in the PL regime were acquired by extracting values of the peak energy from Lorentzian curve fits to the single-QD $\mu$-PL spectra of $\mathrm{X}^{+}$ for known values of $V_{b}$ (Ref. 17). As a result of the QCSE, a quadratic curve defined by $E\left(V_{b}\right)=A V_{b}^{2}+B V_{b}+C$ can be fit to the data points in Fig. 4, yielding a precise relation that can be used to convert between $V_{b}$ and $\mathrm{X}^{+}$transition energy for this particular QD and device (Fig. 4). Therefore, the $V_{b}$ spectra in Figs. 2(b) and 3 (bottom $x$-axes) can be converted to equivalent energy spectra [Figs. 2(b) and 3, top $x$-axes].

The $\mathrm{X}^{+}$transition energy can be expressed also as a function of $F::^{15,18}$

$$
E(F)=E(0)+p F+\beta F^{2},
$$

where $E(0)$ is the $\mathrm{X}^{+}$transition energy at $F=0, p$ is the permanent dipole moment of $\mathrm{X}^{+}$in the vertical (growth) direction, and $\beta$ is the polarizability of $\mathrm{X}^{+}$in the vertical direction. As shown in Fig. 4, fitting this model to the experimental data yields precise values for $p, \beta$, and $E(0)$ of $\mathrm{X}^{+}$.

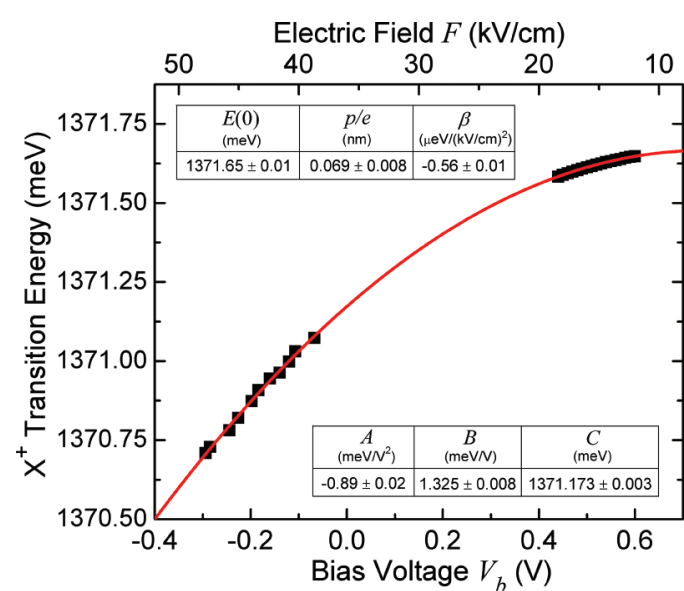

FIG. 4. (Color online) Plot of the $\mathrm{X}^{+}$transition energy as a function of $V_{b}$ (bottom $x$-axis) obtained through both PC and $\mu$-PL spectroscopy. $E\left(V_{b}\right)=A V_{b}^{2}+B V_{b}+C$ can be fit to the experimental data (solid line), yielding a precise relation that is used to convert between $V_{b}$ and $\mathrm{X}^{+}$transition energy. This allows the $V_{b}$ spectra in Figs. 2(b) and 3 (bottom $x$-axes) to be converted to equivalent energy spectra [Figs. 2(b) and 3, top $x$-axes]. $E(F)=E(0)+p F+\beta F^{2}$ can also be fit to the experimental data (top $x$-axis), yielding precise values for $p, \beta$, and $E(0)$.

\section{CONCLUSIONS}

We have demonstrated high-resolution PC spectroscopy of $\mathrm{X}^{+}$in a single InAs/GaAs self-assembled QD. Taking advantage of a Coulomb-mediated renormalization of the $\mathrm{X}^{+}$ transition energy, our approach allows for sub- $\mu \mathrm{eV}$ resolution, which is more than four orders of magnitude higher than that in other approaches. ${ }^{11,12} \mathrm{In}$ addition, this high-resolution $\mathrm{X}^{+}$ spectroscopy affords us the ability to determine precise values for the $\mathrm{X}^{+}$permanent dipole moment and polarizability, by fitting a theoretical model to the measured transition energy as a function of electric field. Optical initialization, manipulation, and readout of a QD spin use the trion as an intermediate state. Therefore, while hole spins should be more favorable as qubits compared to electron spins since their hyperfine interaction with the nuclear spin ensemble leading to decoherence is greatly suppressed, ${ }^{19}$ such high-resolution measurements of the $\mathrm{X}^{+}$spectrum and its dipole moment (i.e., Rabi frequency) are essential requirements for achieving QC operations on QD spin qubits with high fidelity.

\section{ACKNOWLEDGMENTS}

J.D.M. gratefully acknowledges financial support from HEFCE (ORS Award), CCT (CC Scholarship), and NSERC (PGS M, PGS D).
*Corresponding author: jm585@ cam.ac.uk

${ }^{1}$ D. Loss and D. P. DiVincenzo, Phys. Rev. A 57, 120 (1998).

${ }^{2}$ A. Imamoglu, D. D. Awschalom, G. Burkard, D. P. DiVincenzo,

D. Loss, M. Sherwin, and A. Small, Phys. Rev. Lett. 83, 4204 (1999).
${ }^{3}$ D. D. Awschalom, D. Loss, and N. Samarth (eds.), Semiconductor Spintronics and Quantum Computation (Springer, Berlin, 2002).

${ }^{4}$ M. Atatüre, J. Dreiser, A. Badolato, A. Högele, K. Karrai, and A. Imamoglu, Science 312, 551 (2006). 
${ }^{5}$ B. D. Gerardot, D. Brunner, P. A. Dalgarno, P. Öhberg, S. Seidl, M. Kroner, K. Karrai, N. G. Stoltz, P. M. Petroff, and R. J. Warburton, Nature (London) 451, 441 (2008).

${ }^{6}$ J. Berezovsky, M. H. Mikkelsen, N. G. Stoltz, L. A. Coldren, and D. D. Awschalom, Science 320, 349 (2008).

${ }^{7}$ D. Press, T. D. Ladd, B. Zhang, and Y. Yamamoto, Nature (London) 456, 218 (2008).

${ }^{8}$ K. De Greve, P. L. McMahon, D. Press, T. D. Ladd, D. Bisping, C. Schneider, M. Kamp, L. Worschech, S. Höfling, A. Forchel, and Y. Yamamoto, Nat. Phys. 7, 872 (2011).

${ }^{9}$ J. Berezovsky, M. H. Mikkelsen, O. Gywat, N. G. Stoltz, L. A. Coldren, and D. D. Awschalom, Science 314, 1916 (2006).

${ }^{10}$ M. Atatüre, J. Dreiser, A. Badolato, and A. Imamoglu, Nat. Phys. 3, 101 (2007).

${ }^{11}$ A. J. Ramsay, S. J. Boyle, R. S. Kolodka, J. B. B. Oliveira, J. Skiba-Szymanska, H. Y. Liu, M. Hopkinson, A. M. Fox, and M. S. Skolnick, Phys. Rev. Lett. 100, 197401 (2008).
${ }^{12}$ T. M. Godden, J. H. Quilter, A. J. Ramsay, Y. Wu, P. Brereton, S. J. Boyle, I. J. Luxmoore, J. Puebla-Nunez, A. M. Fox, and M. S. Skolnick, Phys. Rev. Lett. 108, 017402 (2012).

${ }^{13}$ K. Ploog, J. Cryst. Growth 81, 304 (1987).

${ }^{14}$ L. M. R. Scolfaro, R. P. Camata, J. M. V. Martins, and J. R. Leite, Superlattices Microstruct. 12, 203 (1992).

${ }^{15}$ J. D. Mar, X. L. Xu, J. J. Baumberg, F. S. F. Brossard, A. C. Irvine, C. Stanley, and D. A. Williams, Phys. Rev. B 83, 075306 (2011).

${ }^{16}$ J. D. Mar, X. L. Xu, J. J. Baumberg, A. C. Irvine, C. Stanley, and D. A. Williams, Appl. Phys. Lett. 99, 031102 (2011).

${ }^{17}$ J. D. Mar, X. L. Xu, J. J. Baumberg, A. C. Irvine, C. Stanley, and D. A. Williams, J. Appl. Phys. 110, 053110 (2011).

${ }^{18}$ P. W. Fry, I. E. Itskevich, D. J. Mowbray, M. S. Skolnick, J. J. Finley, J. A. Barker, E. P. O’Reilly, L. R. Wilson, I. A. Larkin, P. A. Maksym, M. Hopkinson, M. Al-Khafaji, J. P. R. David, A. G. Cullis, G. Hill, and J. C. Clark, Phys. Rev. Lett. 84, 733 (2000).

${ }^{19}$ D. V. Bulaev and D. Loss, Phys. Rev. Lett. 95, 076805 (2005). 